Journal of Universal Mathematics

Vol.4 No.2 PP.128-139 (2021)

ISSN-2618-5660

DOI: $10.33773 /$ jum.957748

\title{
BLOW-UP RESULTS FOR A VISCOELASTIC PLATE EQUATION WITH DISTRIBUTED DELAY
}

\author{
HAZAL YÜKSEKKAYA AND ERHAN PIŞKIN
}

0000-0002-1863-2909 and 0000-0001-6587-4479

\begin{abstract}
In this paper, we consider a nonlinear viscoelastic plate equation with distributed delay. Under suitable conditions, we obtain the blow-up of solutions with distributed delay and source terms. Time delays often appear in many practical problems such as thermal, economic phenomena, biological, chemical, physical, electrical engineering systems, mechanical applications and medicine.
\end{abstract}

\section{INTRODUCTION}

In this paper, we consider the following viscoelastic plate equation with distributed delay and source terms

$$
\begin{cases}u_{t t}+\Delta^{2} u-\int_{0}^{t} g(t-s) \Delta^{2} u(s) d s+\mu_{1} u_{t} & \\ +\int_{\tau_{1}}^{\tau_{2}}\left|\mu_{2}(q)\right| u_{t}(x, t-q) d q & \\ =b|u|^{p-2} u, & (x, t) \in \Omega \times(0, \infty), \\ u(x, t)=\frac{\partial u(x, t)}{\partial v}=0, & (x, t) \in \Omega \times\left(0, \tau_{2}\right), \\ u_{t}(x,-t)=f_{0}(x, t), & x \in \Omega, \\ u(x, 0)=u_{0}(x), u_{t}(x, 0)=u_{1}(x), & \end{cases}
$$

where $b, \mu_{1}>0, p>2$ and $\tau_{1}, \tau_{2}$ are the time delay with $0 \leq \tau_{1}<\tau_{2}, \mu_{2}$ is an $L^{\infty}$ function, and $g$ is a differentiable function under the assumptions (A1), (A2), and (A3). $v$ is the unit outward normal vector.

Problems about the mathematical behavior of solutions for PDEs with time delay effects have become interesting for many authors mainly because time delays often appear in many practical problems such as thermal, economic phenomena, biological, chemical, physical, electrical engineering systems, mechanical applications and medicine. Moreover, it is well known that delay effects may destroy the stabilizing properties of a well-behaved system. In the literature, there are several examples that illustrate how time delays destabilize some internal or boundary control system [6, 7]. Viscous materials are the opposite of elastic materials that posses the ability to dissipate and store the mechanical energy. The mechanical

Date: Received: 2021-06-25; Accepted: 2021-07-30.

2000 Mathematics Subject Classification. 35B44; 35L05, 93D20.

Key words and phrases. Blow-up, Distributed delay, Viscoelastic plate equation. 
properties of these viscous substances are of great importance when they seem in many applications of natural sciences [3].

In 1986, Datko et al. [5] indicated that delay is a source of instability. In [12], Nicaise and Pignotti considered the following wave equation with a linear damping and delay term

$$
u_{t t}-\Delta u+\mu_{1} u_{t}(x, t)+\mu_{2} u_{t}(x, t-\tau)=0 .
$$

They obtained some stability results in the case $0<\mu_{2}<\mu_{1}$. In the absence of delay, Zuazua [26] looked into exponentially stability for the equation (1.2).

Cavalcanti et al. [2], studied the model as follows:

$$
u_{t t}+\gamma \Delta u_{t t}+\Delta^{2} u-\int_{0}^{t} g(t-s) \Delta^{2} u(s) d s+a(t) u_{t}=0,
$$

in $\Omega \times(0, \infty)$, where $a(t)$ is a nonlocal nonlinearity type function. They established the exponential decay result when $\gamma=0$, of the energy in general domains of (1.3). Rivera et al. [25], coupled (1.3) with a dynamic boundary condition and indicated that the sum of the first and second energies decay polynomially and exponentially, according as the relaxation function $g$ decays polynomially or exponentially. Also, for more results on (1.3), see also Lagnese [8].

Mukiawa [9], considered the viscoelastic plate equation as follows

$$
u_{t t}+\Delta^{2} u-\int_{0}^{t} g(t-s) \Delta^{2} u(s) d s+\mu_{1} u_{t}+\mu_{2} u_{t}(t-\tau)=0,
$$

with a constant time delay and partially hinged boundary condition. The author proved a general decay result of the equation (1.4).

In [10], Mustafa and Kafini studied the infinite memory-type plate equation in the presence of constant time delay as follows

$$
u_{t t}+\Delta^{2} u-\int_{0}^{\infty} g(s) \Delta^{2} u(t-s) d s+\mu_{1} u_{t}+\mu_{2} u_{t}(t-\tau)=u|u|^{\gamma} .
$$

The authors proved an explicit and general decay result for the energy, under the condition that $\left|\mu_{2}\right| \leq \mu_{1}$, without restrictive assumptions on the behavior of the relaxation function $g$ at infinity of the equation (1.5).

In [3], Choucha et al. considered the following equation

$$
\begin{aligned}
& u_{t t}-\Delta u-\omega \Delta u_{t}+\int_{0}^{t} g(t-s) \Delta u(s) d s \\
& +\mu_{1} u_{t}+\int_{\tau_{1}}^{\tau_{2}}\left|\mu_{2}(\rho)\right| u_{t}(x, t-\rho) d \rho=b|u|^{p-2} u .
\end{aligned}
$$

The authors obtained the blow-up of solutions under appropriate conditions of the equation (1.6). In [4], the authors showed the exponential growth of solution for the equation (1.6).

The authors obtained the blow-up of solutions under appropriate conditions of the equation (1.6). In [4], the authors showed the exponential growth of solution for the equation (1.6). In recent years, some other authors investigate hyperbolic type equations (see $[13,14,15,16,17,18,19,20,21,22,23,24]$ ).

In this paper, we consider the nonlinear viscoelastic plate equation (1.1) with distributed delay $\left(\int_{\tau_{1}}^{\tau_{2}}\left|\mu_{2}(q)\right| u_{t}(x, t-q) d q\right)$ and source $\left(b|u|^{p-2} u\right)$ terms. Our aim is to get the blow-up results under appropriate conditions for the problem (1.1).

The paper is organized as follows: In section 2, we give some materials that will be used later. In section 3, we state and prove our main result. 


\section{Preliminaries}

In this part, we prepare some materials for the proof of our result. As usual, the notation $\|.\|_{p}$ denotes $L^{p}$ norm, and (.,.) is the $L^{2}$ inner product. In particular, we write $\|\cdot\|$ instead of $\|\cdot\|_{2}$.

Now, we give some assumptions used later:

(A1) $g: R_{+} \rightarrow R_{+}$is a decreasing and differentiable function satisfies

$$
g(t) \geq 0,1-\int_{0}^{\infty} g(s) d s=l>0 .
$$

(A2) There exists a constant $\xi>0$ such that

$$
g^{\prime}(t) \leq-\xi g(t), t \geq 0 .
$$

(A3) $\mu_{2}:\left[\tau_{1}, \tau_{2}\right] \rightarrow R$ is an $L^{\infty}$ function such that

$$
\left(\frac{2 \delta-1}{2}\right) \int_{\tau_{1}}^{\tau_{2}}\left|\mu_{2}(q)\right| d q \leq \mu_{1}, \delta>\frac{1}{2} .
$$

Let $B_{p}>0$ be the constant satisfying [1]

$$
\|\nabla v\|_{p} \leq B_{p}\|\Delta v\|_{p}, \text { for } v \in H_{0}^{2}(\Omega) .
$$

It holds

$$
\begin{aligned}
& \int_{0}^{t} g(t-s)\left(\Delta u(s), \Delta u_{t}(t)\right) d s \\
& =-\frac{1}{2} g(t)\|\Delta u(t)\|^{2}+\frac{1}{2}\left(g^{\prime} o \Delta u\right)(t) \\
& -\frac{1}{2} \frac{d}{d t}\left[(g o \Delta u)(t)-\left(\int_{0}^{t} g(s) d s\right)\|\Delta u(t)\|^{2}\right]
\end{aligned}
$$

where

$$
(g o \Delta u)(t)=\int_{\Omega} \int_{0}^{t} g(t-s)|\Delta u(t)-\Delta u(s)|^{2} d s .
$$

Firstly, similar to [11], we introduce the new variable

$$
y(x, \rho, q, t)=u_{t}(x, t-q \rho),
$$

thus, we get

$$
\left\{\begin{array}{c}
q y_{t}(x, \rho, q, t)+y_{\rho}(x, \rho, q, t)=0 \\
y(x, 0, q, t)=u_{t}(x, t)
\end{array}\right.
$$

Hence, problem (1.1) is equivalent to:

$$
\left\{\begin{array}{c}
u_{t t}+\Delta^{2} u-\int_{0}^{t} g(t-s) \Delta^{2} u(s) d s \\
+\mu_{1} u_{t}+\int_{\tau_{1}}^{\tau_{2}}\left|\mu_{2}(q)\right||y(x, 1, q, t)| d q \quad x \in \Omega, t>0 \\
=b|u|^{p-2} u \\
q y_{t}(x, \rho, q, t)+y_{\rho}(x, \rho, q, t)=0,
\end{array}\right.
$$

with initial and boundary conditions

$$
\left\{\begin{array}{l}
u(x, t)=\frac{\partial u(x, t)}{\partial v}=0, \quad x \in \partial \Omega \\
y(x, \rho, q, 0)=f_{0}(x, q \rho), \\
u(x, 0)=u_{0}(x), u_{t}(x, 0)=u_{1}(x)
\end{array}\right.
$$

where

$$
(x, \rho, q, t) \in \Omega \times(0,1) \times\left(\tau_{1}, \tau_{2}\right) \times(0, \infty) .
$$


Theorem 2.1. Suppose that (2.1), (2.2) and (2.3) hold. Let

$$
\left\{\begin{array}{c}
p \geq 2, n=1,2,3,4 \\
2<p<\frac{2(n-2)}{n-4}, n \geq 5 .
\end{array}\right.
$$

Thus, for any initial data

$$
\left(u_{0}, u_{1}, f_{0}\right) \in H_{0}^{2}(\Omega) \times H_{0}^{2}(\Omega) \times L^{2}\left(\Omega \times(0,1) \times\left(\tau_{1}, \tau_{2}\right)\right),
$$

the problem (2.8)-(2.9) has a unique solution

$$
u \in C\left([0, T] ; H_{0}^{2}(\Omega)\right),
$$

for some $T>0$.

Now, we define the energy functional as follows:

Lemma 2.2. Assume that (2.1), (2.2), (2.3) and (2.10) hold. Let $u$ be a solution of (2.8)-(2.9). Then, $E(t)$ is nonincreasing, such that

$$
\begin{aligned}
E(t)= & \frac{1}{2}\left\|u_{t}\right\|^{2}+\frac{1}{2}\left(1-\int_{0}^{t} g(s) d s\right)\|\Delta u\|^{2}+\frac{1}{2}(g o \Delta u)(t) \\
& +\frac{1}{2} \int_{\Omega} \int_{0}^{1} \int_{\tau_{1}}^{\tau_{2}} q\left|\mu_{2}(q)\right|\left|y^{2}(x, \rho, q, t)\right| d q d \rho d x-\frac{b}{p}\|u\|_{p}^{p},
\end{aligned}
$$

which satisfies

$$
E^{\prime}(t) \leq-c_{1}\left(\left\|u_{t}\right\|^{2}+\int_{\Omega} \int_{\tau_{1}}^{\tau_{2}}\left|\mu_{2}(q)\right|\left|y^{2}(x, 1, q, t)\right| d q d x\right) .
$$

Proof. By multiplying the first equation of (2.8) by $u_{t}$ and integrating over $\Omega$, we obtain

$$
\begin{gathered}
\frac{d}{d t}\left\{\begin{array}{c}
\frac{1}{2}\left\|u_{t}\right\|^{2}+\frac{1}{2}\left(1-\int_{0}^{t} g(s) d s\right)\|\Delta u\|^{2} \\
+\frac{1}{2}(g o \Delta u)(t)-\frac{b}{p}\|u\|_{p}^{p}
\end{array}\right\} \\
=-\mu_{1}\left\|u_{t}\right\|^{2}-\int_{\Omega} u_{t} \int_{\tau_{1}}^{\tau_{2}}\left|\mu_{2}(q)\right||y(x, 1, q, t)| d q d x \\
+\frac{1}{2}\left(g^{\prime} o \Delta u\right)(t)-\frac{1}{2} g(t)\|\Delta u\|^{2}
\end{gathered}
$$

and

$$
\begin{aligned}
\frac{d}{d t} \frac{1}{2} \int_{\Omega} \int_{0}^{1} \int_{\tau_{1}}^{\tau_{2}} q\left|\mu_{2}(q)\right|\left|y^{2}(x, \rho, q, t)\right| d q d \rho d x \\
\quad=-\frac{1}{2} \int_{\Omega} \int_{0}^{1} \int_{\tau_{1}}^{\tau_{2}} 2\left|\mu_{2}(q)\right| y y_{\rho} d q d \rho d x \\
\quad=\frac{1}{2} \int_{\Omega} \int_{\tau_{1}}^{\tau_{2}}\left|\mu_{2}(q)\right|\left|y^{2}(x, 0, q, t)\right| d q d x \\
\quad-\frac{1}{2} \int_{\Omega} \int_{\tau_{1}}^{\tau_{2}}\left|\mu_{2}(q)\right|\left|y^{2}(x, 1, q, t)\right| d q d x \\
\quad=\frac{1}{2}\left(\int_{\tau_{1}}^{\tau_{2}}\left|\mu_{2}(q)\right| d q\right)\left\|u_{t}\right\|^{2} \\
\quad-\frac{1}{2} \int_{\Omega} \int_{\tau_{1}}^{\tau_{2}}\left|\mu_{2}(q)\right|\left|y^{2}(x, 1, q, t)\right| d q d x .
\end{aligned}
$$


Thus,

$$
\begin{aligned}
\frac{d}{d t} E(t)= & -\mu_{1}\left\|u_{t}\right\|^{2}-\int_{\Omega} \int_{\tau_{1}}^{\tau_{2}}\left|\mu_{2}(q)\right|\left|u_{t} y(x, 1, q, t)\right| d q d x \\
& -\frac{1}{2} g(t)\|\Delta u\|^{2}+\frac{1}{2}\left(\int_{\tau_{1}}^{\tau_{2}}\left|\mu_{2}(q)\right| d q\right)\left\|u_{t}\right\|^{2} \\
& +\frac{1}{2}\left(g^{\prime} o \Delta u\right)(t)-\frac{1}{2} \int_{\Omega}^{\tau_{2}} \int_{\tau_{1}}^{\tau_{2}}\left|\mu_{2}(q)\right|\left|y^{2}(x, 1, q, t)\right| d q d x
\end{aligned}
$$

By using (2.13) and (2.14), we obtain (2.11). Utilizing Young's inequality, (2.1), (2.2), (2.3) and (2.15), we get (2.12). Consequently, the proof is completed.

Lemma 2.3. [3] There exists $c>0$, depending on $\Omega$ only, such that

$$
\left(\int_{\Omega}|u|^{p} d x\right)^{s / p} \leq c\left[\|\nabla u\|^{2}+\|u\|_{p}^{p}\right]
$$

for all $u \in L^{p+1}(\Omega)$ and $2 \leq s \leq p$.

From above lemma and by using Sobolev Embedding theorem, we have the following corollary:

Corollary 2.3.1. There exists $c>0$, depending on $\Omega$ only, such that

$$
\left(\int_{\Omega}|u|^{p} d x\right)^{s / p} \leq c\left[\|\Delta u\|^{2}+\|u\|_{p}^{p}\right],
$$

for all $u \in L^{p+1}(\Omega)$ and $2 \leq s \leq p$.

Using the fact that $\|u\|_{2}^{2} \leq c\|u\|_{p}^{2} \leq c\left(\|u\|_{p}^{p}\right)^{2 / p}$, we have the following corollary:

Corollary 2.3.2. There exists $C>0$, depending on $\Omega$ only, such that

$$
\|u\|_{2}^{2} \leq c\left[\|\Delta u\|_{2}^{4 / p}+\left(\|u\|_{p}^{p}\right)^{2 / p}\right] .
$$

Lemma 2.4. [3] There exists $C>0$, depending on $\Omega$ only, such that

$$
\|u\|_{p}^{s} \leq C\left[\|\nabla u\|^{2}+\|u\|_{p}^{p}\right]
$$

for all $u \in L^{p+1}(\Omega)$ and $2 \leq s \leq p$.

From above lemma and by using Sobolev Embedding theorem, we have the following corollary:

Corollary 2.4.1. There exists $C>0$, depending on $\Omega$ only, such that

$$
\|u\|_{p}^{s} \leq c\left[\|\Delta u\|^{2}+\|u\|_{p}^{p}\right]
$$

for all $u \in L^{p+1}(\Omega)$ and $2 \leq s \leq p$.

Now, we define the functional as follows:

$$
\begin{aligned}
H(t)= & -E(t) \\
= & \frac{b}{p}\|u\|_{p}^{p}-\frac{1}{2}\left\|u_{t}\right\|^{2}-\frac{1}{2}\left(1-\int_{0}^{t} g(s) d s\right)\|\Delta u\|^{2} \\
& -\frac{1}{2}(g \circ \Delta u)(t)-\frac{1}{2} \int_{\Omega} \int_{0}^{1} \int_{\tau_{1}}^{\tau_{2}} q\left|\mu_{2}(q)\right|\left|y^{2}(x, \rho, q, t)\right| d q d \rho d x .
\end{aligned}
$$




\section{BlOW-UP RESUlts}

In this part, we establish the blow-up of solutions for the problem (2.8)-(2.9).

Theorem 3.1. Suppose that (2.1)-(2.3) and (2.10) hold. Suppose further that $E(0)<0$ holds. Then, the solution of the problem (2.8)-(2.9) blows up in finite time.

Proof. By (2.11), we have

$$
E(t) \leq E(0) \leq 0
$$

Hence,

$$
\begin{aligned}
H^{\prime}(t) & =-E^{\prime}(t) \geq c_{1}\left(\left\|u_{t}\right\|^{2}+\int_{\Omega} \int_{\tau_{1}}^{\tau_{2}}\left|\mu_{2}(q)\right|\left|y^{2}(x, 1, q, t)\right| d q d x\right) \\
& \geq c_{1} \int_{\Omega} \int_{\tau_{1}}^{\tau_{2}}\left|\mu_{2}(q)\right|\left|y^{2}(x, 1, q, t)\right| d q d x \geq 0,
\end{aligned}
$$

and

$$
0 \leq H(0) \leq H(t) \leq \frac{b}{p}\|u\|_{p}^{p}
$$

Set

$$
\mathcal{K}(t)=H^{1-\alpha}(t)+\varepsilon \int_{\Omega} u u_{t} d x+\frac{\varepsilon \mu_{1}}{2} \int_{\Omega} u^{2} d x,
$$

here $\varepsilon>0$ to be specified later and

$$
\frac{2(p-2)}{p^{2}}<\alpha<\frac{p-2}{2 p}<1
$$

We multiply the first equation of (2.8) by $u$ and with a derivative of (3.4), we obtain

$$
\begin{aligned}
\mathcal{K}^{\prime}(t)= & (1-\alpha) H^{-\alpha}(t) H^{\prime}(t) \\
& +\varepsilon\left\|u_{t}\right\|^{2}+\varepsilon \int_{\Omega} \Delta u \int_{0}^{t} g(t-s) \Delta u(s) d s d x \\
& -\varepsilon\|\Delta u\|^{2}+\varepsilon b \int_{\Omega}|u|^{p} d x \\
& -\varepsilon \int_{\Omega} \int_{\tau_{1}}^{\tau_{2}}\left|\mu_{2}(q)\right||u y(x, 1, q, t)| d q d x .
\end{aligned}
$$

By using

$$
\begin{aligned}
& \varepsilon \int_{\Omega} \int_{\tau_{1}}^{\tau_{2}}\left|\mu_{2}(q)\right||u y(x, 1, q, t)| d q d x \\
\leq & \varepsilon\left\{\delta_{1}\left(\int_{\tau_{1}}^{\tau_{2}}\left|\mu_{2}(q)\right| d q\right)\|u\|^{2}\right. \\
& \left.+\frac{1}{4 \delta_{1}} \int_{\Omega} \int_{\tau_{1}}^{\tau_{2}}\left|\mu_{2}(q)\right|\left|y^{2}(x, 1, q, t)\right| d q d x\right\},
\end{aligned}
$$


and

$$
\begin{aligned}
& \varepsilon \int_{0}^{t} g(t-s) d s \int_{\Omega} \Delta u \Delta u(s) d x d s \\
= & \varepsilon \int_{0}^{t} g(t-s) d s \int_{\Omega} \Delta u(\Delta u(s)-\Delta u(t)) d x d s \\
& +\varepsilon \int_{0}^{t} g(s) d s\|\Delta u\|^{2} \\
\geq & \frac{\varepsilon}{2} \int_{0}^{t} g(s) d s\|\Delta u\|^{2}-\frac{\varepsilon}{2}(g o \Delta u)(t),
\end{aligned}
$$

combining with (3.6), we get

$$
\begin{aligned}
\mathcal{K}^{\prime}(t) \geq & (1-\alpha) H^{-\alpha}(t) H^{\prime}(t)+\varepsilon\left\|u_{t}\right\|^{2} \\
& -\varepsilon\left(1-\frac{1}{2} \int_{0}^{t} g(s) d s\right)\|\Delta u\|^{2} \\
& +\varepsilon b\|u\|_{p}^{p}-\varepsilon \delta_{1}\left(\int_{\tau_{1}}^{\tau_{2}}\left|\mu_{2}(q)\right| d q\right)\|u\|^{2} \\
& -\frac{\varepsilon}{4 \delta_{1}} \int_{\Omega} \int_{\tau_{1}}^{\tau_{2}}\left|\mu_{2}(q)\right|\left|y^{2}(x, 1, q, t)\right| d q d x \\
& +\frac{\varepsilon}{2}(g o \Delta u)(t) .
\end{aligned}
$$

By using (3.2) and setting $\delta_{1}$ such that, $\frac{1}{4 \delta_{1} c_{1}}=\kappa H^{-\alpha}(t)$, we obtain

$$
\begin{aligned}
\mathcal{K}^{\prime}(t) \geq & {[(1-\alpha)-\varepsilon \kappa] H^{-\alpha}(t) H^{\prime}(t)+\varepsilon\left\|u_{t}\right\|^{2} } \\
& -\varepsilon\left[\left(1-\frac{1}{2} \int_{0}^{t} g(s) d s\right)\right]\|\Delta u\|^{2}+\varepsilon b\|u\|_{p}^{p} \\
& -\varepsilon \frac{H^{\alpha}(t)}{4 c_{1} \kappa}\left(\int_{\tau_{1}}^{\tau_{2}}\left|\mu_{2}(q)\right| d q\right)\|u\|^{2}+\frac{\varepsilon}{2}(g o \Delta u)(t) .
\end{aligned}
$$

From (2.21), for $0<a<1$

$$
\begin{aligned}
\varepsilon b\|u\|_{p}^{p}= & \varepsilon p(1-a) H(t)+\frac{\varepsilon p(1-a)}{2}\left\|u_{t}\right\|^{2}+\varepsilon b a\|u\|_{p}^{p} \\
& +\frac{\varepsilon p(1-a)}{2}\left(1-\int_{0}^{t} g(s) d s\right)\|\Delta u\|^{2} \\
& +\frac{\varepsilon}{2} p(1-a)(g o \Delta u)(t) \\
& +\frac{\varepsilon p(1-a)}{2} \int_{\Omega} \int_{0}^{1} \int_{\tau_{1}}^{\tau_{2}} q\left|\mu_{2}(q)\right|\left|y^{2}(x, \rho, q, t)\right| d q d \rho d x
\end{aligned}
$$


with (3.10) implies

$$
\begin{aligned}
\mathcal{K}^{\prime}(t) \geq & {[(1-\alpha)-\varepsilon \kappa] H^{-\alpha}(t) H^{\prime}(t)+\varepsilon\left[\frac{p(1-a)}{2}+1\right]\left\|u_{t}\right\|^{2} } \\
& +\varepsilon\left[\left(\frac{p(1-a)}{2}\right)\left(1-\int_{0}^{t} g(s) d s\right)-\left(1-\frac{1}{2} \int_{0}^{t} g(s) d s\right)\right]\|\Delta u\|^{2} \\
& -\varepsilon \frac{H^{\alpha}(t)}{4 c_{1} \kappa}\left(\int_{\tau_{1}}^{\tau_{2}}\left|\mu_{2}(q)\right| d q\right)\|u\|^{2}+\varepsilon p(1-a) H(t)+\varepsilon b a\|u\|_{p}^{p} \\
& +\frac{\varepsilon p(1-a)}{2} \int_{\Omega} \int_{0}^{1} \int_{\tau_{1}}^{\tau_{2}} q\left|\mu_{2}(q)\right|\left|y^{2}(x, \rho, q, t)\right| d q d \rho d x \\
& +\frac{\varepsilon}{2}(p(1-a)+1)(g o \Delta u)(t) .
\end{aligned}
$$

By using (2.18), (3.3) and Young's inequality, we obtain

$$
\begin{aligned}
H^{\alpha}(t)\|u\|_{2}^{2} & \leq\left(b \int_{\Omega}|u|^{p} d x\right)^{\alpha}\|u\|_{2}^{2} \\
& \leq c\left\{\left(\int_{\Omega}|u|^{p} d x\right)^{\alpha+2 / p}+\left(\int_{\Omega}|u|^{p} d x\right)^{\alpha}\|\Delta u\|_{2}^{4 / p}\right\} \\
& \leq c\left\{\left(\int_{\Omega}|u|^{p} d x\right)^{(p \alpha+2) / p}+\|\Delta u\|_{2}^{2}+\left(\int_{\Omega}|u|^{p} d x\right)^{p \alpha /(p-2)}\right\} .
\end{aligned}
$$

By exploiting (3.5), we obtain

$$
2<\alpha p+2 \leq p \text { and } 2<\frac{\alpha p^{2}}{p-2} \leq p .
$$

Consequently, by Lemma 2.2

$$
H^{\alpha}(t)\|u\|_{2}^{2} \leq c\left(\|u\|_{p}^{p}+\|\Delta u\|_{2}^{2}\right) .
$$

By combining (3.12) and (3.14), we have

$$
\begin{aligned}
\mathcal{K}^{\prime}(t) \geq & {[(1-\alpha)-\varepsilon \kappa] H^{-\alpha}(t) H^{\prime}(t) } \\
& +\varepsilon\left[\frac{p(1-a)}{2}+1\right]\left\|u_{t}\right\|^{2}+\frac{\varepsilon}{2}(p(1-a)+1)(g o \Delta u)(t) \\
& +\varepsilon\left\{\left(\frac{p(1-a)}{2}-1\right)-\int_{0}^{t} g(s) d s\left(\frac{p(1-a)-1}{2}\right)\right. \\
& \left.-\frac{c}{4 c_{1} \kappa}\left(\int_{\tau_{1}}^{\tau_{2}}\left|\mu_{2}(q)\right| d q\right)\right\}\|\Delta u\|^{2} \\
& +\varepsilon\left[a b-\frac{c}{4 c_{1} \kappa}\left(\int_{\tau_{1}}^{\tau_{2}}\left|\mu_{2}(q)\right| d q\right)\right]\|u\|_{p}^{p}+\varepsilon p(1-a) H(t) \\
& +\frac{\varepsilon p(1-a)}{2} \int_{\Omega} \int_{0}^{1} \int_{\tau_{1}}^{\tau_{2}} q\left|\mu_{2}(q)\right|\left|y^{2}(x, \rho, q, t)\right| d q d \rho d x
\end{aligned}
$$

Taking $a>0$ small enough such that

$$
\alpha_{1}=\frac{p(1-a)}{2}-1>0
$$


and suppose

$$
\int_{0}^{\infty} g(s) d s<\frac{\frac{p(1-a)}{2}-1}{\left(\frac{p(1-a)}{2}-\frac{1}{2}\right)}=\frac{2 \alpha_{1}}{2 \alpha_{1}+1} .
$$

Choosing $\kappa$ such that,

$$
\begin{aligned}
\alpha_{2}= & \left(\frac{p(1-a)}{2}-1\right)-\int_{0}^{t} g(s) d s\left(\frac{p(1-a)-1}{2}\right) \\
& -\frac{c}{4 c_{1} \kappa}\left(\int_{\tau_{1}}^{\tau_{2}}\left|\mu_{2}(q)\right| d q\right) \\
> & 0
\end{aligned}
$$

and

$$
\alpha_{3}=a b-\frac{c}{4 c_{1} \kappa}\left(\int_{\tau_{1}}^{\tau_{2}}\left|\mu_{2}(q)\right| d q\right)>0 .
$$

Fixing $\kappa$ and $a$, we have $\varepsilon$ small enough

$$
\alpha_{4}=(1-\alpha)-\varepsilon \kappa>0 .
$$

Hence, for some $\beta>0$, (3.15) becomes

$$
\begin{aligned}
\mathcal{K}^{\prime}(t) \geq & \beta\left\{H(t)+\left\|u_{t}\right\|^{2}+\|\Delta u\|^{2}+\left(g_{o} \Delta u\right)(t)+\|u\|_{p}^{p}\right. \\
& \left.+\int_{\Omega} \int_{0}^{1} \int_{\tau_{1}}^{\tau_{2}} q\left|\mu_{2}(q)\right|\left|y^{2}(x, \rho, q, t)\right| d q d \rho d x\right\} .
\end{aligned}
$$

Thus, it follows that

$$
\mathcal{K}(t) \geq \mathcal{K}(0)>0, t>0
$$

Now, utilizing Holder's and Young's inequalities, we obtain

$$
\begin{aligned}
\|u\|_{2} & =\left(\int_{\Omega} u^{2} d x\right)^{\frac{1}{2}} \\
& \leq\left[\left(\int_{\Omega}\left(|u|^{2}\right)^{p / 2} d x\right)^{\frac{2}{p}}\left(\int_{\Omega} 1 d x\right)^{1-\frac{2}{p}}\right]^{\frac{1}{2}} \\
\leq & C\|u\|_{p}
\end{aligned}
$$

and

$$
\left|\int_{\Omega} u u_{t} d x\right| \leq\left\|u_{t}\right\|_{2}\|u\|_{2} \leq c\left\|u_{t}\right\|_{2}\|u\|_{p} .
$$

Therefore,

$$
\begin{aligned}
\left|\int_{\Omega} u u_{t} d x\right|^{\frac{1}{1-\alpha}} & \leq c\left\|u_{t}\right\|_{2}^{\frac{1}{1-\alpha}}\|u\|_{p}^{\frac{1}{1-\alpha}} \\
& \leq c\left[\left\|u_{t}\right\|_{2}^{\frac{\theta}{1-\alpha}}+\|u\|_{p}^{\frac{\mu}{1-\alpha}}\right],
\end{aligned}
$$

here $\frac{1}{\mu}+\frac{1}{\theta}=1$. Taking $\theta=2(1-\alpha)$, we obtain

$$
\frac{\mu}{1-\alpha}=\frac{2}{1-2 \alpha} \leq p \text {. }
$$


For $s=\frac{2}{(1-2 \alpha)}$, we get

$$
\left|\int_{\Omega} u u_{t} d x\right|^{\frac{1}{1-\alpha}} \leq c\left(\left\|u_{t}\right\|_{2}^{2}+\|u\|_{p}^{s}\right) .
$$

Hence, Corollary 2.3.1 gives that

$$
\begin{aligned}
\left|\int_{\Omega} u u_{t} d x\right|^{\frac{1}{1-\alpha}} & \leq c\left[\left\|u_{t}\right\|_{2}^{2}+\|u\|_{p}^{p}+\|\Delta u\|_{2}^{2}\right] \\
& \leq c\left[\left\|u_{t}\right\|_{2}^{2}+\|u\|_{p}^{p}+\|\Delta u\|_{2}^{2}+(g o \Delta u)(t)\right] .
\end{aligned}
$$

Therefore,

$$
\begin{aligned}
\mathcal{K}^{\frac{1}{1-\alpha}}(t) & =\left(H(t)^{1-\alpha}+\varepsilon \int_{\Omega} u u_{t} d x+\frac{\varepsilon \mu_{1}}{2} \int_{\Omega} u^{2} d x\right)^{\frac{1}{1-\alpha}} \\
& \leq c\left[H(t)+\left|\int_{\Omega} u u_{t} d x\right|^{\frac{1}{1-\alpha}}+\|u\|_{2}^{\frac{2}{1-\alpha}}+\|\Delta u\|_{2}^{\frac{2}{1-\alpha}}\right] \\
& \leq c\left[H(t)+\left\|u_{t}\right\|^{2}+\|u\|_{p}^{p}+\|\Delta u\|^{2}+(g o \Delta u)(t)\right] .
\end{aligned}
$$

By (3.17) and (3.22), we get

$$
\mathcal{K}^{\prime}(t) \geq \lambda \mathcal{K}^{\frac{1}{1-\alpha}}(t),
$$

here $\lambda>0$, which depends on $\beta$ and $c$. An integration of (3.23), we have

$$
\mathcal{K}^{\frac{\alpha}{1-\alpha}}(t) \geq \frac{1}{\mathcal{K}^{\frac{-\alpha}{1-\alpha}}(0)-\lambda \frac{\alpha}{(1-\alpha)} t} .
$$

Thus, $\mathcal{K}(t)$ blows up in a finite time

$$
T \leq T^{*}=\frac{1-\alpha}{\lambda \alpha \mathcal{K}^{\alpha /(1-\alpha)}(0)}
$$

As a result, we complete the proof.

\section{Conclusion}

In recent years, there has been published much work concerning the wave equations (Kirchhoff, Petrovsky, Bessel,... etc.) with different state of delay time (constant delay, time-varying delay,... etc.). However, to the best of our knowledge, there were no blow-up results for the nonlinear viscoelastic plate equation with

distributed delay. We have been obtained the blow-up of solutions with distributed delay and source terms under suitable conditions.

\section{ACKnowledgments}

The authors would like to thank the reviewers and editors of Journal of Universal Mathematics.

\section{Funding}

The authors are grateful to DUBAP (ZGEF.20.009) for research funds.

The Declaration of Conflict of Interest/ Common Interest

The author(s) declared that no conflict of interest or common interest 


\section{The Declaration of Ethics Committee Approval}

This study does not be necessary ethical committee permission or any special permission.

\section{The Declaration of Research and Publication Ethics}

The author(s) declared that they comply with the scientific, ethical, and citation rules of Journal of Universal Mathematics in all processes of the study and that they do not make any falsification on the data collected. Besides, the author(s) declared that Journal of Universal Mathematics and its editorial board have no responsibility for any ethical violations that may be encountered and this study has not been evaluated in any academic publication environment other than Journal of Universal Mathematics.

\section{REFERENCES}

[1] R.A. Adams, J.J.F. Fournier, Sobolev Spaces, Academic Press, (2003).

[2] M.M. Cavalcanti, V.N. Domingos Cavalcanti and J. Ferreira, Existence and uniform decay for nonlinear viscoelastic equation with strong damping, Math. Methods Appl. Sci., 24, pp.10431053 (2001).

[3] A. Choucha, D. Ouchenane and S. Boulaaras, Blow-up of a nonlinear viscoelastic wave equation with distributed delay combined with strong damping and source terms, J. Nonlinear Funct. Anal., 2020,pp.1-10 (2020).

[4] A. Choucha, D. Ouchenane and K. Zennir, Exponential growth of solution with $L_{p}$-norm for class of non-linear viscoelastic wave equation with distributed delay term for large initial data, Open J. Math. Anal., 3(1), pp.76-83 (2020).

[5] R. Datko, J. Lagnese and M.P. Polis, An example on the effect of time delays in boundary feedback stabilization of wave equations, SICON, 24(1), pp.152-156 (1986).

[6] J.K. Hale, S.M. Verduyn Lunel, Introduction to Functional-Differential Equations, Appl. Math. Sci., 99, x+447, (Springer-Verlag, New York),(1993).

[7] M. Kafini, S.A. Messaoudi, A blow-up result in a nonlinear wave equation with delay, Mediterr. J. Math., 13, pp.237-247 (2016).

[8] J.E. Lagnese, Asymptotic energy estimate for Kirchhoff plates subject to weak viscoelastic damping, Internat. Ser. of Numer. Math., 91, Birkhcauser-Verlag, Bassel, (1989).

[9] S.E. Mukiawa, Decay result for a delay viscoelastic plate equation, Bull. Braz. Math. Soc. New Ser., 51, pp.333-356 (2020).

[10] M.I. Mustafa, M. Kafini, Decay rates for memory-type plate system with delay and source term, Math. Meth. Appl. Sci., 40(4),pp. 883-895 (2017).

[11] S. Nicaise, C. Pignotti, Stabilization of the wave equation with boundary or internal distributed delay, Differ. Integral Equ., 21, pp.935-958 (2008).

[12] S. Nicaise, C. Pignotti, Stability and instability results of the wave equation with a delay term in the boundary or internal feedbacks, SIAM J. Control Optim, 45(5), pp.1561-1585 (2006).

[13] E. Pişkin, H. Yüksekkaya, Local existence and blow up of solutions for a logarithmic nonlinear viscoelastic wave equation with delay, Comput. Methods Differ. Equ., 9(2), pp.623-636 (2021).

[14] E. Pişkin, H. Yüksekkaya, Nonexistence of global solutions of a delayed wave equation with variable-exponents, Miskolc Math. Notes, pp.1-19. (Accepted)

[15] E. Pişkin, H. Yüksekkaya, Blow up of solution for a viscoelastic wave equation with mLaplacian and delay terms, Tbil. Math. J., SI (7),pp. 21-32 (2021).

[16] E. Pişkin, H. Yüksekkaya, Blow-up of solutions for a logarithmic quasilinear hyperbolic equation with delay term, J. Math. Anal., 12(1), pp. 56-64 (2021).

[17] E. Pişkin, H. Yüksekkaya, Non-existence of solutions for a Timoshenko equations with weak dissipation, Math. Morav., 22 (2), pp.1-9 (2018).

[18] E. Pişkin and H. Yüksekkaya, Decay of solutions for a nonlinear Petrovsky equation with delay term and variable exponents, The Aligarh Bull. of Maths., 39(2),pp. 63-78,( 2020). 
[19] H. Yüksekkaya, E. Pişkin, Blow up of Solutions for Petrovsky Equation with Delay Term, Journal of Nepal Mathematical Society.4 (1),pp.76-84 (2021).

[20] E. Pişkin, H. Yüksekkaya, Mathematical behavior of the solutions of a class of hyperbolic-type equation, J. BAUN Inst. Sci. Technol.20(3), pp.117-128(2018).

[21] H. Yüksekkaya, E. Pişkin, S.M. Boulaaras, B.B. Cherif and S.A. Zubair, Existence, Nonexistence, and Stability of Solutions for a Delayed Plate Equation with the Logarithmic Source, Adv. Math. Phys.,2021, pp.1-11, (2021), https://doi.org/10.1155/2021/8561626.

[22] E. Pişkin, H. Yüksekkaya and N. Mezouar, Growth of Solutions for a Coupled Viscoelastic Kirchhoff System with Distributed Delay Terms, Menemui Matematik (Discovering Mathematics) 43(1),pp. 26-38, (2021).

[23] E. Pişkin, H. Yüksekkaya, Global Attractors for the Higher-Order Evolution Equation, AMNS, 5(1), pp.195-210 (2020).

[24] E. Pişkin and N. Polat, On the decay of solutions for a nonlinear Petrovsky equation, Math. Sci. Lett., 3(1), pp.43-47 (2013).

[25] J.E. Rivera, E.C. Lapa and R. Barreto, Decay rates for viscoelastic plates with memory, J. Elast., 44(1), pp.61-87 (1996).

[26] E. Zuazua, Exponential decay for the semi-linear wave equation with locally disributed damping, Commun. Part. Diff. Eq., 15, pp.205-235 (1990).

(Hazal Yüksekkaya (Corresponding Author)) Dicle University, Department of Mathematics, Diyarbakir, Turkey

Email address: hazally.kaya@gmail.com

(Erhan Pişkin) Dicle University, Department of Mathematics, Diyarbakir, Turkey

Email address: episkin@dicle.edu.tr 